\title{
Tuberculosis screening in Portuguese healthcare workers using the tuberculin skin test and the interferon- $\gamma$ release assay
}

\author{
J. Torres Costa*,\#, , R. Sá*, M.J. Cardoso ${ }^{\text {, }+, ~ R . ~ S i l v a *, \#, ף, ~ J . ~ F e r r e i r a *, ~ C . ~ R i b e i r o *, ~}$ \\ M. Miranda*,\#,ף, J.L. Plácido**\# and A. Nienhaus ${ }^{\S}$
}

ABSTRACT: The prevalence of latent tuberculosis (TB) infection (LTBI) and the incidence of active tuberculosis in healthcare workers (HCWs) in a Portuguese hospital were examined.

This cross-sectional study comprises 4,735 hospital workers screened between May 2005 and September 2008. Tuberculin skin test (TST) and interferon- $\gamma$ release assay (IGRA) were used simultaneously in 1,219 HCWs (25.7\%). Radiographs were taken in symptomatic HCWs or in testpositive HCWs. The tests were repeated annually or bi-annually depending on risk assessment.

IGRA was positive in $32.6 \%$ and TST in $74.2 \%$ of the HCWs. Years spent in healthcare were a risk factor for a positive IGRA, but not for a positive TST. Repeated bacillus Calmette-Guérin vaccination increased the probability of TST+/IGRA- discordance $(35.4 \%$ versus $54.4 \%$, respectively). In those tested three times with TST during the study period $(n=59)$, the mean diameter of TST increased from 5 to 7 to $10 \mathrm{~mm}$. Within $3 \mathrm{yrs}, 31 \mathrm{HCWs}$ were diagnosed with active TB (annual incidence rate 191 out of 100,000 people). In eight HCWs with active TB, TST and IGRA were performed at the time of diagnosis and each test was positive.

TB burden in HCWs in Portugal is high. With IGRA, the number of radiographs needed to exclude active TB could have been reduced by about half without missing a case of active TB. Therefore IGRA should be introduced into TB screening programmes.

KEYWORDS: Healthcare workers, interferon- $\gamma$ release assay, Portugal, tuberculin skin test, tuberculosis

creening healthcare workers (HCWs) for latent tuberculosis infection (LTBI) and active tuberculosis (TB) is fundamental in infection control programmes in hospitals [1]. In Portugal, screening of HCWs for TB has been performed only in the context of contact tracing through the public health department, while occupational medicine has been nonexistent in most Portuguese hospitals. Since 2005, Hospital S. João, in Northern Portugal (Porto), has been building up an Occupational Health Division for the hospital staff, which screens HCWs for TB on a regular basis, depending on risk assessment following Centers for Disease Control and Prevention guidelines [1].

For about a century, the tuberculin skin test (TST) has been used to detect LTBI. However, the TST has known limitations, including cross-reactivity with bacillus Calmette-Guérin (BCG) and nontubercular mycobacteria (NTM) infections [2]. Advances in molecular biology have led to the development of new in vitro assays that measure interferon (IFN)- $\gamma$ released by sensitised $\mathrm{T}$ cells after stimulation with Mycobacterium tuberculosis antigens. These tests are more specific than the TST because they use antigens not shared by any of the BCG vaccine strains nor by the more common species of NTM (e.g. Mycobacterium avium) [3]. Besides a higher specificity and an at-least equal sensitivity as the TST, interferon- $\gamma$ release assays (IGRAs) correlate better with surrogate measures of exposure to $M$. tuberculosis [4-6] and have a higher predictive value for LTBI progression to active TB in close contact in lowincidence settings [7].

So far, only a few systematic investigations of LTBI in HCWs using the IGRA have been published [8-14]. Therefore, we studied the prevalence of LTBI and active TB in Portuguese HCWs and compared IGRA and TST performance.

\section{MATERIALS AND METHODS}

\section{Study setting and study subjects}

The population of this cross-sectional study comprises all workers of the Hospital S. João who participated in TB screening from May 2005
AFFILIATIONS

*Occupational Health Division, Alameda Professor Hernâni Monteiro \#Allergy and Clinical Immunology Division, Alameda Professor Hernâni Monteiro

${ }^{+}$Clinical Pathology Division, Alameda Professor Hernâni Monteiro Hospital S. João

'Faculty of Medicine, Alameda Professor Hernâni Monteiro, Porto University, Porto, Portugal.

${ }^{\S}$ Institution for Statutory Accident Insurance and Prevention in the Health and Welfare Services, Hamburg, Germany.

CORRESPONDENCE

A. Nienhaus

Institution for Statutory Accident Insurance and Prevention in the Health and Welfare Services Pappelallee 35-37 D-22089 Hamburg Germany E-mail: albert.nienhaus@bgwonline.de

Received:

April 012009

Accepted after revision: May 282009 


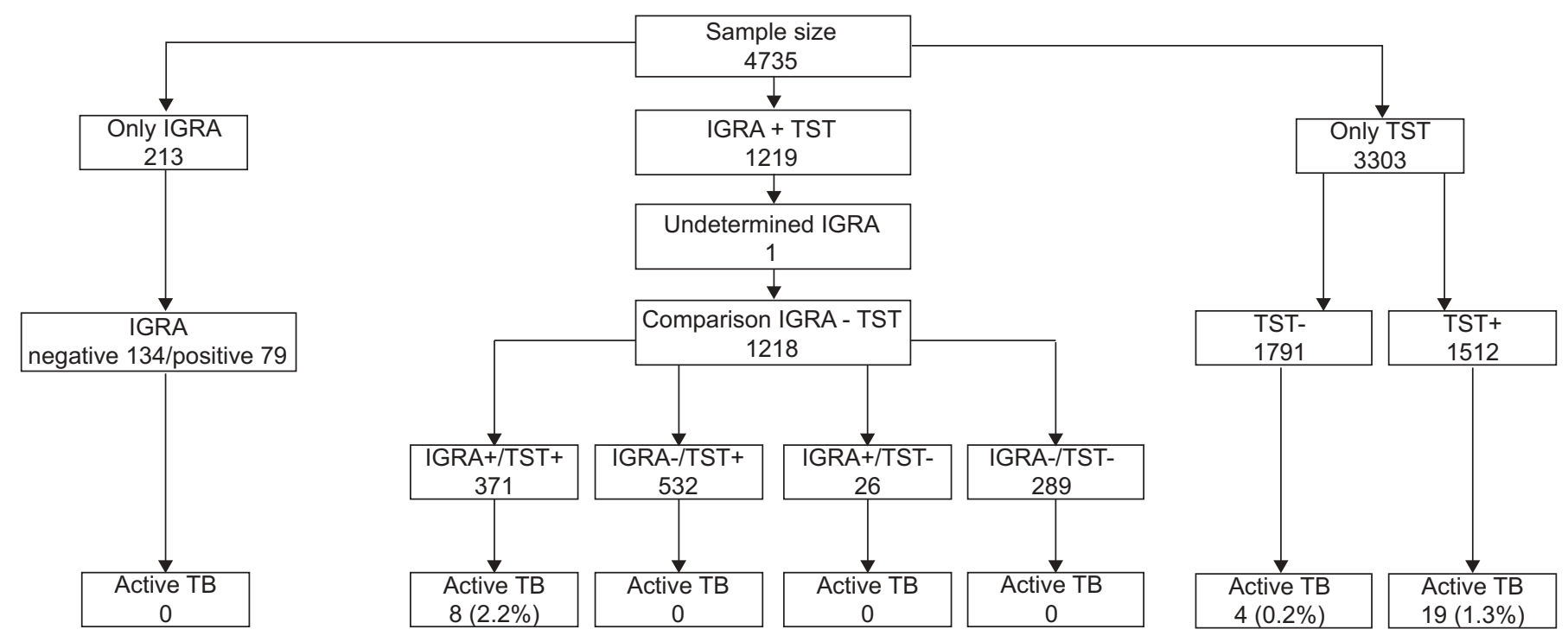

FIGURE 1. Study population. IGRA: interferon- $\gamma$ release assay; TST: tuberculin skin test; TB: tuberculosis.

until September 2008. HCWs in infection and TB wards are considered to be at high risk, workers with regular patient contact in other wards are considered to be at medium risk and workers with no regular patient contact or no contact with biological material are considered to be at low risk [1]. Upon commencement of employment, all workers are examined. HCWs considered at high risk are evaluated annually. All others are evaluated every other year or after known exposure to patients with active TB.

Screening was initially performed using TST only. Since January 2007, TST and IGRA have been performed simultaneously. TST was performed when the diameter of a previous TST was $<15 \mathrm{~mm}$ or when no previous TST result was known. A chest radiograph was performed when TST was $\geqslant 10 \mathrm{~mm}$ or in HCWs with TB symptoms.

BCG vaccination was assessed through the individual vaccination register or by scars. Following the national vaccination plan, BCG vaccination for newborns is mandatory in Portugal and, until January 2000, was repeated if TST was $<5 \mathrm{~mm}$ [15]. Therefore, every HCW has been vaccinated at least once.

TST was performed by trained personnel following standard procedures. In brief, $0.1 \mathrm{~mL}$ (2 TU) of purified protein derivate (RT23; Statens Serum Institute, Copenhagen, Denmark) was injected intradermally at the volar side of the forearm and the transverse diameter of the induration was read 72-96 h later. A diameter $\geqslant 10 \mathrm{~mm}$ was considered positive.

Before TST application, an interview was performed and blood for the IGRA was drawn. As IGRA, the QuantiFERON-TB $\mathbb{R}$ Gold In-Tube Assay (Cellestis Limited, Carnegie, Australia) was administrated, following the manufacturer's protocol. Observers were blinded to the results of the TST and vice versa.

\section{Statistical analysis}

Chi-squared tests were used for categorical data. Adjusted OR and $95 \%$ CI were calculated for putative predictive variables using conditional logistic regression. Model building was performed backwards using the chance criteria for variable selection [16].

All persons gave their informed consent prior to their inclusion in the study.

\section{RESULTS}

The study population comprised 4,735 HCWs. In 213 HCWs $(4.5 \%)$, only IGRA results were available because the TST was contraindicated, refused, or the second appointment for TST reading was missed (fig. 1). The characteristics of those 1,219 HCWs (25.7\%) tested simultaneously with TST and IGRA are given in table 1 .

TST was positive in $74.2 \%$ and IGRA in $32.6 \%$ of the HCWs. The probability of a positive IGRA increased with the induration diameter of the TST (table 2), with age and with years spent as an HCW (table 3 ). The OR for TST $\geqslant 10 \mathrm{~mm}$ in HCWs with three or more additional BCG vaccinations after birth was 2.0 (95\% CI 1.29-3.02). The variable "years since the last vaccination" did not influence TST results. A positive IGRA was less likely when only $\leqslant 10$ yrs had passed since the last vaccination (OR 0.2; 95\% CI 0.09-0.52). No association with profession or risk assessment was observed in either test.

TST+/IGRA- discordance increased with the number of BCG vaccinations (table 4). TST+/IGRA- discordance was $40.5 \%$ when the last vaccination was $>15$ yrs ago and $56.3 \%$ when $1-$ 10 yrs had passed. TST+/IGRA- discordance in nurses was higher than in other professions (table 4) and thay had TST more often repeated during the study period (30.5\%) than, for example, physicians (14.7\%). Risk assessment was inversely correlated with positive concordant results (low risk 38.7\%, high risk $26.7 \%$ ). Age $>60$ yrs was associated with increased TST-/IGRA+ discordance (table 4).

In a subgroup of $59 \mathrm{HCWs}$ in whom the TST was repeated three times during the study period (time between the test: 


\begin{tabular}{|c|c|c|}
\hline \multirow[t]{2}{*}{ TABLE 1} & \multirow[t]{2}{*}{ com } & \multirow[t]{2}{*}{ eron- $\gamma$} \\
\hline & & \\
\hline \multicolumn{3}{|l|}{ Age } \\
\hline $16-29$ yrs & 443 & 36.4 \\
\hline 30-39 yrs & 336 & 27.6 \\
\hline $40-49$ yrs & 212 & 17.4 \\
\hline $50-59$ yrs & 199 & 16.3 \\
\hline$\geqslant 60 \mathrm{yrs}$ & 28 & 2.3 \\
\hline \multicolumn{3}{|l|}{ Sex } \\
\hline Female & 878 & 72.1 \\
\hline Male & 340 & 27.9 \\
\hline \multicolumn{3}{|l|}{ BCG vaccination } \\
\hline Only at birth & 370 & 30.4 \\
\hline 1 additional & 426 & 35.0 \\
\hline 2 additional & 301 & 24.7 \\
\hline$\geqslant 3$ additional & 121 & 9.9 \\
\hline \multicolumn{3}{|l|}{ Years since last $B C G$} \\
\hline$>15 \mathrm{yrs}$ & 920 & 75.5 \\
\hline$>10-15$ yrs & 227 & 18.6 \\
\hline $1-10$ years & 71 & 5.8 \\
\hline \multicolumn{3}{|l|}{ Profession } \\
\hline Administrator & 186 & 15.3 \\
\hline Auxiliaries, cleaning staff & 188 & 15.4 \\
\hline Technicians (radiology, lab, etc.) & 85 & 7.0 \\
\hline Nurses & 548 & 45.0 \\
\hline Physicians & 211 & 17.3 \\
\hline \multicolumn{3}{|l|}{ Risk assessment } \\
\hline Low risk & 173 & 14.2 \\
\hline Moderate risk & 407 & 33.4 \\
\hline High risk & 638 & 52.4 \\
\hline \multicolumn{3}{|l|}{ Time working in healthcare } \\
\hline Start of work & 208 & 17.1 \\
\hline$<5$ yrs & 301 & 27.7 \\
\hline $5-<10$ yrs & 212 & 17.4 \\
\hline $10-<15$ yrs & 142 & 11.7 \\
\hline $15-<20$ yrs & 90 & 7.4 \\
\hline$\geqslant 20$ yrs & 265 & 21.8 \\
\hline
\end{tabular}

12 months), the median of the induration diameter increased from $5 \mathrm{~mm}$ via $7 \mathrm{~mm}$ to $10 \mathrm{~mm}$ (fig. 2).

Active tuberculosis was diagnosed in 31 HCWs (fig. 1) based on positive PCR $(n=2)$ or on culture-confirmed positive smear $(n=29)$. Assuming a dynamic cohort in which every HCW was present for 41 months (May 2005 through September 2008), the annual incidence rate was 191.6 out of 100,000 people. In eight HCWs with active TB, TST and IGRA were performed simultaneously at the time of diagnosis and both tests were positive.

In $16(51.6 \%)$ out of the $31 \mathrm{HCWs}$ with active TB (fig. 1), the diagnosis was initiated by the Occupational Health Division. 13 presented themselves with typical TB symptoms and three were diagnosed based on positive TST and IGRA followed by a suspicious radiograph.

\begin{tabular}{|c|c|c|c|c|c|c|}
\hline \multirow[t]{4}{*}{ TABLE 2} & \multicolumn{6}{|c|}{$\begin{array}{l}\text { Tuberculin skin test (TST) diameter by } \\
\text { interferon- } \gamma \text { release assay (IGRA) results }\end{array}$} \\
\hline & \multicolumn{6}{|c|}{ IGRA } \\
\hline & \multicolumn{2}{|c|}{ Negative } & \multicolumn{2}{|c|}{ Positive } & \multicolumn{2}{|c|}{ Total } \\
\hline & $\mathbf{n}$ & Row\% & $\mathbf{n}$ & Row\% & $\mathbf{n}$ & Col\% \\
\hline \multicolumn{7}{|l|}{ TST } \\
\hline 0-4 mm & 133 & 97.1 & 4 & 2.9 & 137 & 11.2 \\
\hline $5-9 \mathrm{~mm}$ & 156 & 87.6 & 22 & 12.4 & 178 & 14.6 \\
\hline $10-15 \mathrm{~mm}$ & 341 & 70.6 & 142 & 29.4 & 483 & 39.7 \\
\hline$>15 \mathrm{~mm}$ & 191 & 45.5 & 229 & 54.5 & 420 & 34.5 \\
\hline All & 821 & 67.4 & 397 & 32.6 & 1218 & 100.0 \\
\hline
\end{tabular}

\section{DISCUSSION}

This is the largest study to investigate the performance of the TST and the IGRA when screening HCWs, which allowed for the analysis of risk factors for discordant results of the two tests. The annual incidence rate of active TB in Portuguese HCWs (191.6 per 100,000 people) was about six times higher than the one in the general population in Portugal (32 out of 100,000 people) in 2006 [17] and the prevalence of LTBI varied widely depending on the test used. Therefore, the proportion of HCWs needing radiograph might have been reduced from $74.2 \%$ with positive TST to $32.6 \%$ if only IGRA-positive HCWs undergo radiograph in order to exclude active TB.

So far, little is known about the effect of repeated BCG vaccination on the probability of TST+/IGRA- discordant results $[18,19]$. Repeated BCG vaccination increased the probability of positive TSTs not confirmed by IGRA. Recent BCG vaccination

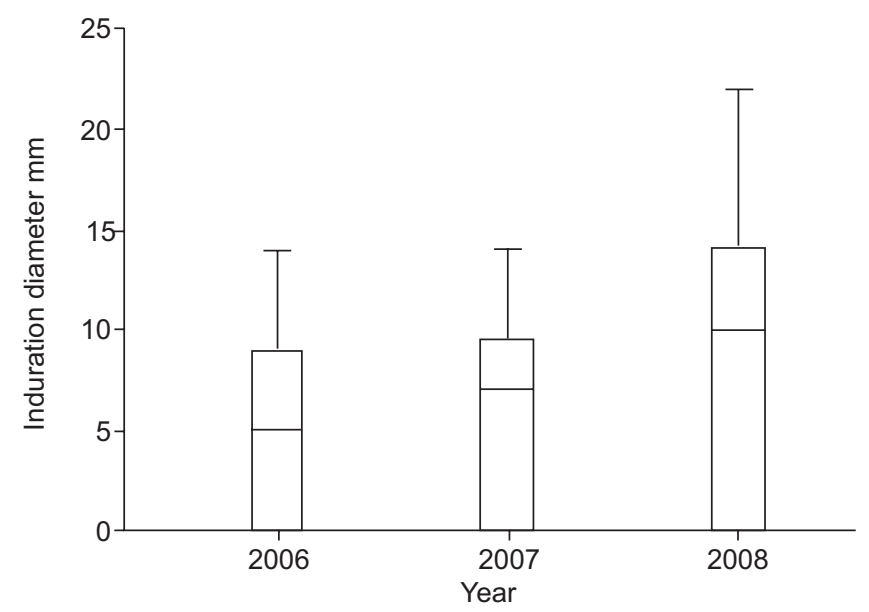

FIGURE 2. Box plot of induration diameter in $\mathrm{mm}$ in 59 healthcare workers with three tuberculin skin tests in 2006, 2007 and 2008. Horizontal bars represent medians, boxes represent interquartile range and whiskers represent the data range. 


\begin{tabular}{|c|c|c|c|c|c|c|}
\hline \multirow[t]{3}{*}{ TABLE 3} & \multirow{2}{*}{\multicolumn{3}{|c|}{$\begin{array}{l}\text { TST) } \geqslant 10 \mathrm{~m} \\
\mathrm{nt} \text { putative ris } \\
\text { TST } \geqslant \mathbf{1 0} \mathbf{m m}\end{array}$}} & \multicolumn{3}{|c|}{ say (IGRA), and the respective } \\
\hline & & & & & A-pos & \\
\hline & n (\%) & OR & $95 \% \mathrm{Cl}$ & n (\%) & OR & $95 \% \mathrm{Cl}$ \\
\hline \multicolumn{7}{|l|}{ Age } \\
\hline $40-49$ yrs & $88(41.5)$ & 1.0 & $0.68-1.38$ & $87(41.0)$ & 1.8 & $1.26-2.70$ \\
\hline $50-59$ yrs & 78 (39.2) & 1.1 & $0.71-1.56$ & $99(49.7)$ & 2.1 & $1.40-3.17$ \\
\hline$\geqslant 60 \mathrm{yrs}$ & $8(28.6)$ & 0.7 & $0.23-1.68$ & $12(42.9)$ & 1.4 & $0.63-3.28$ \\
\hline \multicolumn{7}{|l|}{ Sex } \\
\hline Female & $391(44.5)$ & 1 & & $278(31.7)$ & 1 & \\
\hline 2 additional & 149 (49.5) & 1.6 & $1.18-2.24$ & $74(24.6)$ & 0.8 & $0.55-1.18$ \\
\hline 3-10 additional & $66(54.5)$ & 2.0 & $1.29-3.02$ & $20(16.5)$ & 0.6 & $0.34-1.07$ \\
\hline \multicolumn{7}{|l|}{ Years since last $B C G$} \\
\hline$>15 \mathrm{yrs}$ & $373(40.5)$ & 1 & & 355 (38.6) & 1 & \\
\hline$>10-15$ yrs & $119(52.4)$ & 1.3 & $0.91-1.80$ & $36(15.9)$ & 0.4 & $0.28-0.67$ \\
\hline $1-10 \mathrm{yrs}$ & $40(56.3)$ & 1.4 & $0.84-2.38$ & $6(8.5)$ & 0.2 & $0.09-0.52$ \\
\hline \multicolumn{7}{|l|}{ Profession } \\
\hline Administrator & 76 (40.9) & 1 & & $75(40.3)$ & 1 & \\
\hline Auxiliaries, cleaning staff & 65 (34.6) & 0.7 & $0.49-1.13$ & $76(40.4)$ & 1.5 & $0.95-2.45$ \\
\hline Technicians (radiology, lab, etc.) & $44(51.8)$ & 1.4 & $0.82-2.34$ & $22(25.9)$ & 0.8 & $0.43-1.45$ \\
\hline Nurses & $276(50.4)$ & 1.3 & $0.91-1.82$ & $138(25.2)$ & 0.8 & $0.52-1.21$ \\
\hline \multicolumn{7}{|l|}{ Risk assessment } \\
\hline $5-<10$ yrs & $102(48.1)$ & 1.2 & $0.81-1.83$ & $58(27.4)$ & 1.8 & $1.08-2.86$ \\
\hline $10-<15$ yrs & $65(45.8)$ & 1.1 & $0.73-1.78$ & $52(36.6)$ & 2.9 & $1.34-4.87$ \\
\hline $15-<20$ yrs & $36(40.0)$ & 0.9 & $0.56-1.58$ & 39 (43.3) & 3.0 & $1.68-5.26$ \\
\hline$\geqslant 20 \mathrm{yrs}$ & $104(39.2)$ & 1.2 & $0.79-1.79$ & $125(47.2)$ & 2.6 & $1.64-4.13$ \\
\hline
\end{tabular}

BCG: bacillus Calmette-Guérin

or repeated BCG vaccination decreased the probability of a negative IGRA. This is most likely due to the fact that TSTnegative HCWs are revaccinated, inducing a positive TST without changing the IGRA.

The increase of TST diameter in the small subgroup $(n=59)$ tested three times during the study period most likely indicates a booster effect of serial testing [20] as does the higher rate of TST+/QFT- results in nurses, who also have TST repeated most often. The effect of serial testing with TST on IGRA has still to be elucidated. Preliminary results suggest that IGRA is not substantially influenced when TST is administered 3 days before the IGRA [21].
Working in healthcare is a well-known risk factor for TB [22-24]. As per our data, the probability of a positive IGRA (table 3) increased with years spent in healthcare, while the TST did not reveal such an association. Surprisingly, neither risk assessment [1] nor profession was associated with TST or IGRA. In the two European fingerprint studies [25, 26], the majority of workrelated active TB cases occurred when the infection risk was not suspected and preventive measures were not taken. Rotation of the staff is another explanation for the lack of this association. Furthermore, our cross-sectional design might dilute the expected association.

Screening HCWs for LTBI with TST in our populations had shortcomings. The rate of positive TST was high and influenced 


\begin{tabular}{|c|c|c|c|c|c|c|c|c|c|}
\hline \multirow[t]{4}{*}{ TABLE 4} & $\begin{array}{l}\text { cord } \\
\text { ve ri }\end{array}$ & $\begin{array}{l}\text { aberc } \\
\text { ctors }\end{array}$ & kin & T) $\geqslant$ & and & on- $\gamma$ & ass & RA) $r \in$ & \multirow{4}{*}{$\mathrm{p}$-value } \\
\hline & \multicolumn{8}{|c|}{ TST/IGRA } & \\
\hline & \multicolumn{2}{|c|}{$-/-$} & \multicolumn{2}{|c|}{$+/-$} & \multicolumn{2}{|c|}{$-/+$} & \multicolumn{2}{|c|}{$+/+$} & \\
\hline & $\mathbf{n}$ & $\%$ & $n$ & $\%$ & $\mathbf{n}$ & $\%$ & $\mathbf{n}$ & $\%$ & \\
\hline $16-29 \mathrm{yrs}$ & 132 & 29.8 & 212 & 47.9 & 10 & 2.3 & 89 & 20.1 & \\
\hline 30-39 yrs & 90 & 26.8 & 146 & 43.5 & 6 & 1.8 & 94 & 28.0 & \\
\hline $40-49$ yrs & 37 & 17.5 & 88 & 41.5 & 2 & 0.9 & 85 & 40.1 & \\
\hline $50-59$ yrs & 22 & 11.1 & 78 & 39.2 & 5 & 2.5 & 94 & 47.2 & \\
\hline$\geqslant 60 \mathrm{yrs}$ & 8 & 28.6 & 8 & 28.6 & 3 & 10.7 & 9 & 32.1 & $<0.001$ \\
\hline Only at birth & 73 & 19.7 & 131 & 35.4 & 10 & 2.7 & 156 & 42.2 & \\
\hline 1 additional & 103 & 24.2 & 186 & 43.7 & 10 & 2.3 & 127 & 29.8 & \\
\hline 2 additional & 78 & 25.9 & 149 & 49.5 & 5 & 1.7 & 69 & 22.9 & \\
\hline 3-10 additional & 35 & 28.9 & 66 & 54.5 & 1 & 0.8 & 19 & 15.7 & $<0.001$ \\
\hline \multicolumn{10}{|l|}{ Years since last BCG } \\
\hline$>15 \mathrm{yrs}$ & 192 & 20.9 & 373 & 40.5 & 24 & 2.6 & 331 & 36.0 & \\
\hline$>10-15$ yrs & 72 & 31.7 & 119 & 52.4 & 1 & 0.4 & 35 & 15.4 & \\
\hline $1-10$ years & 25 & 35.2 & 40 & 56.3 & 1 & 1.4 & 5 & 7.0 & $<0.001$ \\
\hline \multicolumn{10}{|l|}{ Profession } \\
\hline Administrator & 35 & 18.8 & 76 & 40.9 & 4 & 2.2 & 71 & 38.2 & \\
\hline Auxiliaries, cleaning staff & 47 & 25.0 & 65 & 34.6 & 4 & 2.1 & 72 & 38.3 & \\
\hline Start of work & 82 & 39.4 & 83 & 39.9 & 2 & 1.0 & 41 & 19.7 & \\
\hline$<5$ yrs & 79 & 26.2 & 142 & 47.2 & 10 & 3.3 & 70 & 23.3 & \\
\hline $5-<10$ yrs & 52 & 24.5 & 102 & 48.1 & 6 & 2.8 & 52 & 24.5 & \\
\hline $10-<15$ yrs & 25 & 17.6 & 65 & 45.8 & 1 & 0.7 & 51 & 35.9 & \\
\hline $15-<20$ yrs & 15 & 16.7 & 36 & 40.0 & 1 & 1.1 & 38 & 42.2 & \\
\hline$\geqslant 20 \mathrm{yrs}$ & 36 & 13.6 & 104 & 39.2 & 6 & 2.3 & 119 & 44.9 & $<0.001$ \\
\hline
\end{tabular}

BCG: bacillus Calmette-Guérin. ${ }^{*}$ : repeated TST during study period: nurses (30.5\%) physicians (14.7\%), p-value <0.0005.

by repeated BCG vaccination, allowing little discrimination between HCWs at risk of having or of progressing to active TB. Therefore, our data corroborate the conclusion of a Taiwan HCW study [27] that the TST is not useful in contact investigation among BCG-vaccinated HCWs. In the subgroup undergoing TST and IGRA simultaneously, all eight HCWs diagnosed with active TB were positive in both tests. Restricting radiographs to symptomatic or IGRA-positive HCWs would have reduced the number of radiographs by more than half (TST $74.2 \%$ versus IGRA $32.6 \%$ ) without decreasing the number of active TB cases detected in our population.

\section{STATEMENT OF INTEREST}

None declared.

\section{REFERENCES}

1 Jensen PA, Lambert LA, Iademarco MF, et al. Guidelines for preventing the transmission of Mycobacterium tuberculosis in Healthcare Settings, 2005. MMWR 2005; 54: 1-141.

2 Menzies D. What does tuberculin reactivity after Bacille CalmetteGuerin vaccination tell us? Clin Infect Dis 31, 2000: Suppl. 3, S71-S74. 
3 Andersen P, Munk ME, Pollock JM, et al. Specific immune-based diagnosis of tuberculosis. Lancet 2000; 356: 1099-1104.

4 Nahid P, Pai M, Hopewell PC. Advances in the diagnosis and treatment of tuberculosis. Proc Am Thorac Soc 2006; 3: 103-110.

5 Pai M, Riley LW, Colford JM. Interferon- $\gamma$ assays in the immunodiagnosis of tuberculosis: a systematic review. Lancet Infect Dis 2004; 4: 761-776.

6 Menzies D, Pai M, Comstock G. Meta-analysis: new tests for the diagnosis of latent tuberculosis infection: areas of uncertainty and recommendations for research. Ann Intern Med 2007; 146: 340-352.

7 Diel R, Loddenkemper R, Meywald-Walter K, et al. Predictive value of a whole blood IFN- $\gamma$ assay for the development of active TB disease. Am J Respir Crit Care Med 2008; 177: 1164-1170.

8 Harada N, Nakajima Y, Higuchi K, et al. Screening for tuberculosis infection using whole-blood interferon- $\gamma$ and Mantoux testing among Japanese healthcare workers. Infect Control Hosp Epidemiol 2006; 27: 442-448.

9 Soberg B, Andersen $\mathrm{AB}$, Larsen $\mathrm{HK}$, et al. Detecting a low prevalence of latent tuberculosis among health care workers in Denmark detected by M. tuberculosis specific INF- $\gamma$ whole-blood test. Scand J Infect Dis 2007; 39: 554-559.

10 Kobashi Y, Obase Y, Fukuda M, et al. Usefulness of QuantiFERON TB-2G, a diagnostic method for latent tuberculosis infection, in a contact investigation of health care workers. Intern Med 2007; 46: 1543-1549.

11 Mirtskhulava V, Kempker R, Shields KL, et al. Prevalence and risk factors for latent tuberculosis infection among health care workers in Georgia. Int J Tuberc Lung Dis 2008; 12: 513-519.

12 Nienhaus A, Schablon A, Siano B, et al. Evaluation of the interferongamma release assay in healthcare workers. Int Arch Occup Environ Health 2008; 81: 295-300.

13 Schablon A, Beckmann G, Harling M, et al. Prevalence of latent tuberculosis infection among healthcare workers in a hospital for pulmonary diseases. J Occup Med Toxicol 2009; 4: 1.

14 Vinton $\mathrm{P}$, Mihrshahi S, Johnson $\mathrm{P}$, et al. Comparison of QuantiFERON-TB Gold In-Tube test and tuberculin skin test for identification of latent Mycobacterium tuberculosis infection in healthcare staff and association between positive test results and known risk factors for infection. Infect Control Hosp Epidemiol. 2009; 30: 215-221.
15 National Vaccination Plan: Programa Nacional de Vacinação. Direcção-Geral da Saúde - Ministério da Saúde www.dgsaude.pt/ upload/membro.id/ficheiros/i007442.pdf Last accessed: May 1, 2009.

16 Hosmer D, Lemeshow S. Applied Logistic Regression. New York, John Wiley \& Sons, 2000.

17 WHO Report 2008. Global Tuberculosis Control: Surveillance, Planning, Financing. WHO/HTM/TB/2008.393.

18 Farhat M, Greenaway, Pai M, et al. False-positive tuberculin skin tests: what is the absolute effect of BCG and non-tuberculous mycobacteria? Int J Tuberc Lung Dis 2006; 10: 1192-1204.

19 Nienhaus A, Schablon A, Diel R. Interferon- $\gamma$ release assay for the diagnosis of latent TB infection - analysis of discordant results, when compared to the tuberculin skin test. PLOS ONE 2008; 3: e2665.

20 Menzies D. Interpretation of repeated tuberculin tests. Boosting conversion, and reversion. Am J Respir Crit Care Med 1999; 159: 15-21.

21 Leyten EMS, Prins C, Bossink AWJ, et al. Effect of tuberculin skin testing on a Mycobacterium tuberculosis specific interferon- $\gamma$ assay. Eur Respir J 2007; 29: 1212-1216.

22 Menzies D, Joshi R, Pai M. Risk of tuberculosis infection and disease associated with work in health care settings. Int J Tuberc Lung Dis 2007; 11: 593-605.

23 Seidler A, Nienhaus A, Diel R. Review of epidemiological studies on the occupational risk of tuberculosis in low-incidence areas. Respiration 2005; 72: 431-446.

24 Saleiro S, Santos AR, Vidal O, et al. Tuberculosis in hospital department health care workers. Revista Portuguesa de Pneumologia 2007; 13: 789-799.

25 Diel R, Seidler A, Nienhaus A, et al. Occupational risk of tuberculosis transmission in a low incidence area. Respir Res 2005; 6: 35-45.

26 De Vries G, Sebek MM, Lambregts-van Weezenbeek CS. Healthcare workers with tuberculosis infected during work. Eur Respir J 2006; 28: 1216-1221.

27 Lee S S-J, Liu Y-C, Huang T-S, et al. Comparison of the interferon- $\gamma$ release assay and the tuberculin skin test for contact investigation of tuberculosis in BCG-vaccinated health care workers. Scandinavian Journal of Infectious Disease 2008; 40: 373-380. 